\title{
PET IMAGE RECONSTRUCTION AND DENOISING ON HEXAGONAL LATTICES
}

\author{
Syed Tabish Abbas, Jayanthi Sivaswamy \\ CVIT, IIIT-Hyderabad
}

\begin{abstract}
Nuclear imaging modalities like Positron emission tomography (PET) are characterized by a low SNR value due to the underlying signal generation mechanism. Given the significant role images play in current-day diagnostics, obtaining noise-free PET images is of great interest. With its higher packing density and larger and symmetrical neighbourhood, the hexagonal lattice offers a natural robustness to degradation in signal. Based on this observation, we propose an alternate solution to denoising, namely by changing the sampling lattice. We use filtered back projection for reconstruction, followed by a sparse dictionary based denoising and compare noise-free reconstruction on the Square and Hexagonal lattices. Experiments with PET phantoms (NEMA, Hoffman) and the Shepp-Logan phantom show that the improvement in denoising, post reconstruction, is not only at the qualitative but also quantitative level. The improvement in PSNR in the hexagonal lattice is on an average between 2 to $10 \mathrm{~dB}$. These results establish the potential of the hexagonal lattice for reconstruction from noisy data, in general.
\end{abstract}

Index Terms - PET Images, Denoising, Hexagonal lattice, Image Reconstruction

\section{INTRODUCTION}

Image denoising is a classical problem in image processing. It continues to be an active area of research. A variety of techniques for denoising have been proposed recently based on Non-Local means [1] wavelets [2], curvelets [3], total variation [4] and sparse representation/Dictionary learning [5]. Image denoising is critical in the medical domain where images are typically obtained via a reconstruction process. Depending on the nature of the modality and acquisition methodology, the reconstructed images are corrupted with noise. For instance, the need to minimize exposure (or dosage) levels of a subject to ionizing radiations such as X-ray employed in computed tomography (CT), invariably incurs a low SNR. The quality of the reconstructed image plays a key role in its usefulness as a basis for medical diagnostics. Better image quality naturally facilitates more accurate diagnosis.

In nuclear imaging, the problem is especially acute since the acquired signal is based on low photon counts that result from a radioactive decay process. Due to the randomness involved in the decay process, the noise problem cannot be alleviated by merely improving the sensor mechanism such as employing photo multipliers. Hence, this is handled at the signal processing level. Recently, the low SNR problem has been tackled with compressive sensing (CS) based approaches. CS solutions incorporating sparse constraints have been used both during and post reconstruction. Examples of the former are low dose CT [6] and PET [7] reconstruction with undersampling. Examples of the latter are the deblurring solutions proposed in [8],[9].

In this paper, we argue that there is an alternative avenue for solving the noise problem, namely, by employing the hexagonal sampling lattice and demonstrate a dictionary based approach to denoising of PET images. Hexagonal lattices offer consistent connectivity and superior angular resolution motivating their study for several applications such as edge detection, morphological processing, etc., [10], [11]. The utility of this lattice in reconstruction has not been reported in literature barring a method for CT reconstruction which reports improved efficiency and memory management with hexagonal lattice [12]. Since optical cameras acquire images sampled on a square grid, resampling is required to consider the hexagonal grid-based solutions, thus limiting their practical application. However, this is not the case with PET (or CT) images, as the signal is acquired as a sinogram first thus permitting the choice of the hexagonal lattice more readily for reconstructing and denoising the final image. We choose a sparse dictionary based approach for denoising since it has been shown to perform well on images from natural images [5] as well as MR and fluorescence microscopy images [9]. Our approach does not incorporate the noise model in the dictionary learning step in order to clearly assess the role the change of lattice in PET image denoising using the simplest possible pipeline: reconstruction onto a hexagonal lattice using filtered back projection (FBP) followed by sparse dictionarybased denoising. The paper presents results of assessing the denoising performance across lattices using 3 phantoms, one of which is analytical and the other two being standard phantoms used for PET reconstruction studies: the Shepp-Logan (analytical) NEMA and Hoffman. 


\section{HEXAGONAL IMAGE ADDRESSING}

Hexagonally sampled images are defined on horizontally aligned hexagonal lattices generated using the basis matrix $\mathbf{V}_{h}$ defined as

$$
\mathbf{V}_{h}=\left(h_{1}, h_{2}\right)=\left(\begin{array}{cc}
1 & \frac{1}{2} \\
0 & \frac{\sqrt{3}}{2}
\end{array}\right)
$$

where the columns $h_{i} ; i=1,2$ are the basis vectors of the lattice which are clearly non-orthogonal. Based on the above matrix it is possible to define a neighbourhood $\mathcal{N}$ of a point as the following set.

$$
\mathcal{N}_{h}^{6}=\left\{h_{1}, h_{2}, h_{1}-h_{2}\right\}
$$

It may be noted that $\left|h_{2}-h_{1}\right|=\left|h_{1}\right|=\left|h_{2}\right|$ and hence all the neighbouring sites in a hexagonal 6 neighbourhood are equidistant. These properties distinguish the hexagonal lattice from the square lattice in two key ways: (a) consistent connectivity due to equidistant points in $\mathcal{N}$ and (b) redundancy in the information captured at the lattice points due to the non-orthogonal basis vectors. These two factors can be beneficial to denoising with the hexagonal lattice, relative to a square lattice.

Another implication of the above definition for the basis vectors is that the Cartesian coordinates for the lattice points are irrational which is cumbersome due to floating point errors that may occur during addressing. We hence choose to follow the single-index, base- 7 addressing convention proposed in [10] as it has been shown to be efficient. The indexing scheme uses a positional numbering system based on hierarchical aggregates to address pixels. A sample image with pixel indices is shown in figure 1. This linear indexing helps to define an image patch easily. We define a hexagonal patch of order $n$ centered at location $l_{7}$ as the set of pixels given by:

$$
{ }^{h} \mathcal{P}_{l}^{n}=\left\{l_{7}, l_{7}+1_{7}, l_{7}+2_{7}, \ldots l_{7}+\left(7^{n}\right)_{7}\right\}
$$

Where, $1_{7}, 2_{7}, .$. , is numbers $1,2, .$. to base 7 . For example a patch of order $1(\mathrm{O} 1)$ at location $15_{7}$ is given by:

$$
{ }^{h} \mathcal{P}_{42}^{1}=\{15,16,10,14,2,1,63\}
$$

In general, an $\mathrm{O} n$ patch will have $7^{n}$ elements/pixels. Figure 1 shows and example of a $\mathrm{O} 2$ patch which is stored as a vector. $\mathrm{O} 2$ patch is seen to be similar in structure, to an $\mathrm{O} 1$ patch, with a central $\mathrm{O} 1$ patch surrounded by six $\mathrm{O} 1$ patches. Thus, there is a natural hierarchical structure to the image neighbourhoods.

\section{PROPOSED PIPELINE}

PET is a nuclear imaging modality used to study functional activities of living tissues such as glucose metabolism, etc.

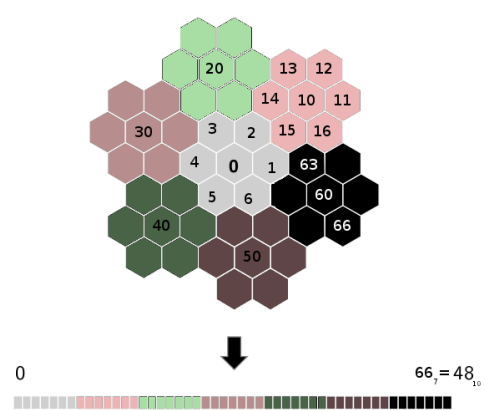

Fig. 1: A hexagonal patch of order 2 and its representation as a vector.

The measured data generally consists of a sinogram and an attenuation profile which is used to correct the sinogram data. The proposed pipeline for denoising has two stages: In stage 1 , the PET image is reconstructed onto a desired lattice from the given sinogram and in stage 2 the reconstructed image is denoised using a sparse dictionary learned from the noisy image. Details of these stages are explained next.

\subsection{Filtered back projection: stage 1}

Filtered back projection (FBP) is an analytical reconstruction method, which essentially is an algorithm for inverting the sinogram or set of projections. It is derived from the Fourier slice theorem which establishes the relationship between the projections and the Fourier transform of the cross section $f(x, y)$ being imaged. Reconstruction involves filtering each of the projections (rows of the sinogram) and backprojecting the filtered results. FBP assumes the data to be noise-free and hence leads to noisy reconstructions given a noisy sinogram. In practice, therefore, iterative, statistical reconstruction methods [13], are employed to achieve a good SNR. However, since our focus in the present work is on assessing the role of lattice, FBP serves as an appropriate baseline algorithm to compare the results.

\subsection{Sparse dictionary based denoising: stage 2}

The denoising is based on the KSVD algorithm [5]. The main steps in the algorithm are: Dictionary learning, sparse coding, reconstruction of the denoised image using sparse code and the learnt dictionary. The denoising is based on the sparsity of the image which means fewer atoms capture the 'clean' signal whereas the noise is captured by many or all atoms. Various methods [14] [15] etc have been proposed for learning sparse dictionaries. We have used the 'online approach' [15] to learn a dictionary which we briefly review next.

Randomly sampled patches from images are vectorized to generate training data for learning the dictionary. In the case of square lattice, the patch ${ }^{s} \mathcal{P}_{l}^{n}$ is vectorized by row-major ordering to obtain a vector $\mathbf{x}_{s} \in \mathcal{R}^{n^{2}}$. For the hexagonal patch 


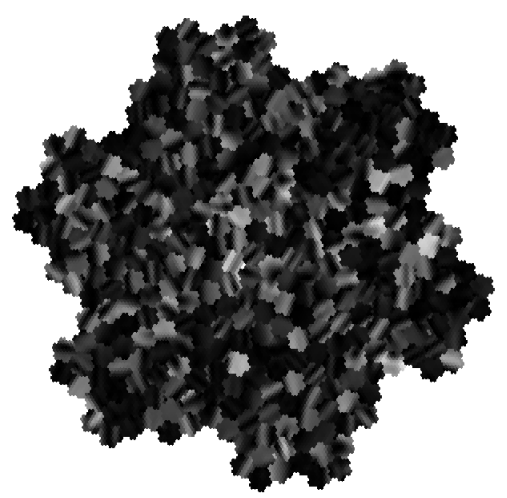

Fig. 2: Sample Dictionary learned over the hexagonal lattice

${ }^{h} \mathcal{P}_{l}^{n}$, the adopted positional indexing method yields a vector directly $\mathbf{x}_{h} \in \mathcal{R}^{7^{n}}$ (see figure 1). For a fair comparison of the lattices, $n$ was chosen to be 2 in hexagonal case and 7 in the square case, to get a 49-dimensional vector for both lattices. The dictionary is learned by solving the following optimization problem.

$$
\begin{gathered}
\min _{D \in \mathcal{C}, \alpha \in \mathcal{R}^{k \times n}} \frac{1}{2}\|\mathbf{X}-\mathbf{D} \alpha\|_{F}^{2}+\lambda\|\alpha\|_{1,1} \\
\mathcal{C}=\left\{\mathbf{D} \in \mathcal{R}^{m \times k} \text { s.t } \forall j=1, \ldots k, d_{j}^{T} d_{j}^{T} \leq 1\right\}
\end{gathered}
$$

where, $\mathbf{X}=\left(x_{s(h) 1}, x_{s(h) 2}, \ldots x_{s(h) n}\right)$ is a matrix of training samples(vectors), $\alpha$ is the sparse code matrix for the vectors. $\|\cdot\|_{F}$ represents the Frobenius norm and $\|\cdot\|_{1,1}$ the $l_{1}$ norm. Two separate dictionaries $\mathbf{D}_{h}$ (hexagonal) and $\mathbf{D}_{s}$ (square) are learnt using (3). This method is fast and optimized for a large training set (which is the case for densely sampled image patches). The regularization parameter $\lambda=0.6$ was used while training the dictionary. An example of individual atoms learned in the hexagonal case is shown in Figure 2.

Sparse coding is done using Cholesky factorization-based orthogonal matching pursuit of the test signals. The algorithm efficiently computes in parallel, the sparse codes $\alpha$ by approximately solving the following (NP-hard) problem.

$$
\min _{\alpha}\|\alpha\|_{0} \text { s.t }\|x-D \alpha\|_{2}^{2} \leq \epsilon
$$

During the denoising stage, densely sampled patches from images are reconstructed using the sparse dictionary learned in previous step. A (sparse code) weighted combination of dictionary atoms are averaged to obtain the final denoised image.

\section{RESULTS}

The denoising method was assessed with 3 standard phantom images: i) Shepp-Logan phantom, which is analytically derived and routinely used to evaluate reconstruction algorithms ii) the NEMA and Hoffman brain phantoms which are specifically used to test PET reconstruction. In order to display the

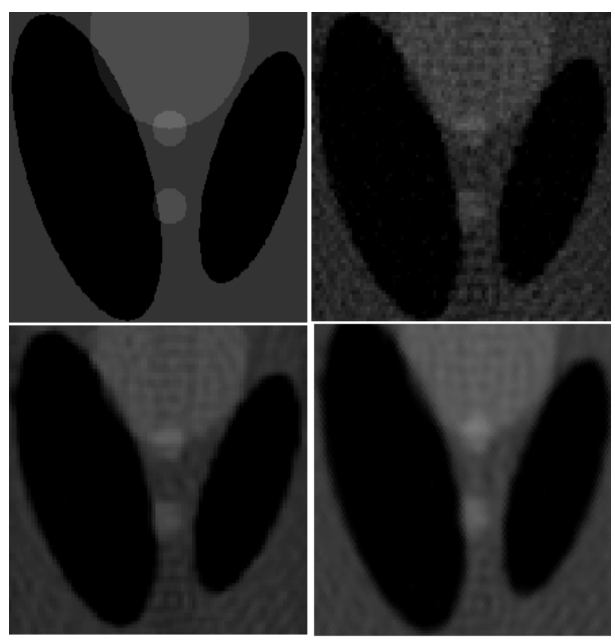

Fig. 3: Comparison of denoising results for the Shepp-Logan phantom. Clockwise from top left: original, noisy image, denoised resuls on hexagonal and square grids

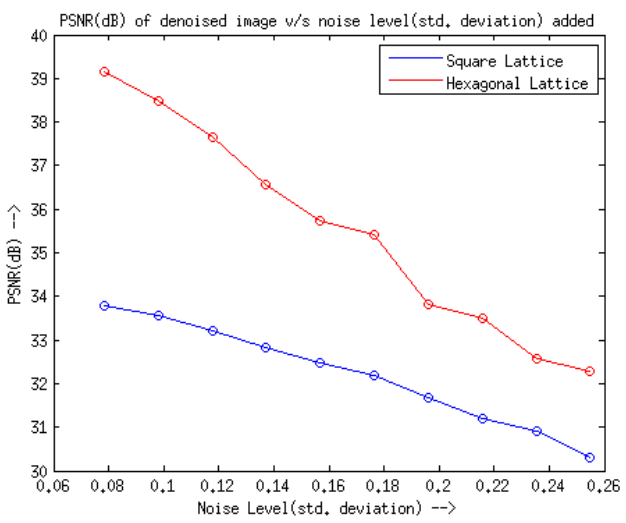

Fig. 4: Average PSNR for the Shepp-Logan phantom for various noise levels

reconstructed results on hexagonal lattice, the pixels were visualized with square hyper-pixels using the code provided in [10]. The Shepp-Logan phantom, unlike the the other two, permits a controlled study of denoising. In our experiments, first, the phantom image $I$ (generated using Matlab) was degraded with additive Gaussian noise to model the noisy source $I_{n}$. Next, the sinogram, constructed by computing the Radon transform of $I_{n}$, was used to reconstruct noisy images $I_{r}$ onto square/hexagonal lattices. Finally, $I_{r}$ was denoised in the native lattices.

The original image $I$, its noisy reconstructions $I_{r}$ and the denoised results are shown in Figure 3 for standard deviation 0.08 . From these results, we see that the central small, white, circle has better definition and shape fidelity on the hexagonal compared to the square lattice. The denoised image on the hexagonal grid is smoother as well.

The denoising was assessed quantitatively by varying the noise and computing the PSNR with $I$ as the 'clean' original. The experiments were repeated 5 times and the average values were recorded. Figure 4 shows this average PSNR 


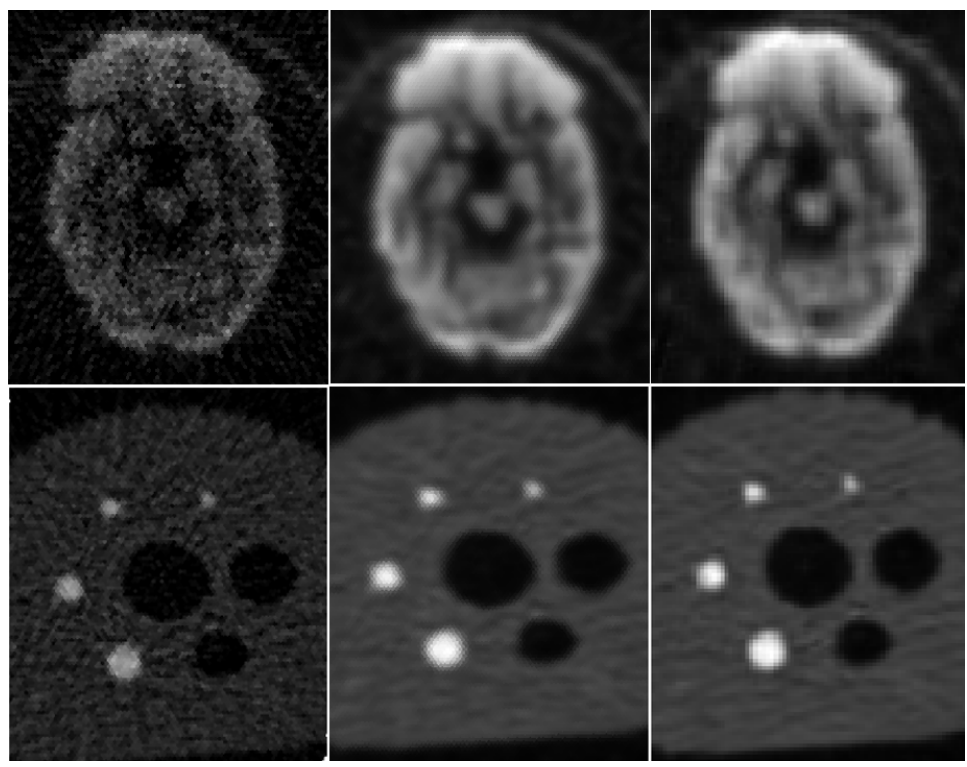

Fig. 5: Comparison of denoising results for NEMA and Hoffman phantoms. Left to right: Noisy reconstruction, denoised images on hexagonal and square lattices.
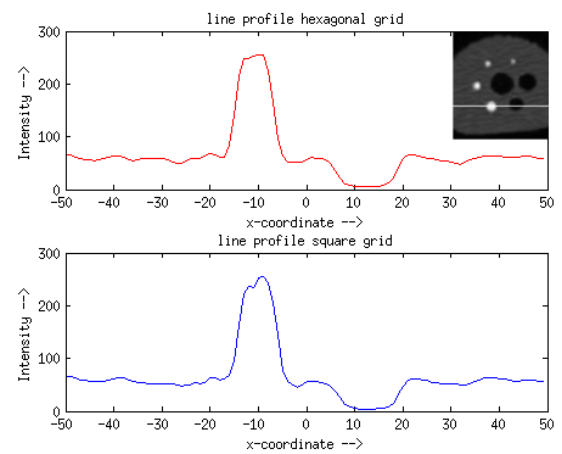

Fig. 6: Scanline comparison for NEMA on Hexagonal (red), Square (blue) lattices. Inset image shows the scan line. The labelled pixel positions in the plots are with the origin at the centre of the image

as a function of noise levels. A trend analysis of the plot shows that, for high PSNR (i.e. low noise levels) a change to hexagonal lattice results in a $5 d B$ improvement in denoising while for high noise levels, the improvement is half as much. Figure 5 shows the noisy reconstructed $\left(I_{r}\right)$ and denoised results ( $I$ for the NEMA and Hoffman phantoms. A quantitative assessment of NEMA phantom was done in two ways: a) An 'inverse' PSNR metric, which treats the denoised image as the clean signal and the noisy reconstruction as the 'noisy signal', was computed. A large magnitude of 'error' indicates good denoising. The average (over 5 repetitions) inverse PSNR for the NEMA phantom were $-59.7 d B$ and $-51.6 d B$ for the square and hexagonal lattices, respectively. For the Hoffman phantom these values were $-51.5 d B$ (square) and $-41.5 d B$ (hexagonal). This demonstrates that the improvement in denoising with hexagonal lattice is between 8 to $10 \mathrm{~dB}$. b) The intensity profile along several scan lines in the denoised im- age were analysed. This was done only for NEMA phantom as it is the standard used for PET calibration. A scan line profile is shown in Figure 6. The line position, as indicated in the inset image, covers two objects of opposite polarity on a noisy background. Hence, the ideal profile should be flat at the location of the objects. This is the case especially for the bright object in the hexagonal lattice whereas it is not in the square lattice. The region between bright and dark objects represent the background which appears noisier in the square case both in Figure 5 and the profile in Figure 6. Thus, the hexagonal lattice appears to be better at preserving the fidelity of the shape after reconstruction and denoising.

\section{CONCLUSION}

In this paper, we argued that an alternate solution for improving image denoising, can be a change in the underlying sampling lattice. We extended the adaptive dictionary based denoising to hexagonally sampled images. The experimental results confirm that using a hexagonal lattice for reconstruction and denoising of PET sinogram data improves the performance of reconstruction both qualitatively as well as quantitatively by as much as $10 d B$. This can be improved further with incorporation of a noise model in the denoising. While the proposed method was proven on PET medical image reconstruction, it is quite possible to extend its applicability to images from other modalities such as low dose CT, spectral CT and ultrasound.

\section{REFERENCES}

[1] A Buades et al., "A review of image denoising algorithms, with a new one," Multiscale Modeling \& Simulation, vol. 4, no. 2, pp. 490-530, 2005. 
[2] S G Chang et al., "Spatially adaptive wavelet thresholding with context modeling for image denoising," IEEE TIP, vol. 9, no. 9, pp. 1522-1531, 2000.

[3] J-L Starck et al., "The curvelet transform for image denoising," IEEE TIP, vol. 11, no. 6, pp. 670-684, 2002.

[4] L I Rudin et al., "Nonlinear total variation based noise removal algorithms," Physica D: Nonlinear Phenomena, vol. 60, no. 1, pp. 259-268, 1992.

[5] M Elad and M Aharon, "Image denoising via sparse and redundant representations over learned dictionaries," IEEE TIP, vol. 15, no. 12, pp. 3736-3745, 2006.

[6] Q Xu et al., "Low-dose x-ray ct reconstruction via dictionary learning," IEEE TMI, vol. 31, no. 9, pp. 1682-1697, 2012.

[7] S Valiollahzadeh et al., "Dictionary learning in compressed sensing using undersampled data in pet imaging," Med. Physics, vol. 40, no. 6, pp. 400-400, 2013.

[8] S Valiollahzadeh et al., "Mo-g-17a-05: Pet image deblurring using adaptive dictionary learning," Medical Physics, vol. 41, no. 6, pp. 437438, 2014.

[9] Liyan Ma, Lionel Moisan, Jian Yu, and Tieyong Zeng, "A dictionary learning approach for poisson image deblurring," IEEE TMI, vol. 32, no. 7, pp. 1277-1289, 2013.

[10] L Middleton and J Sivaswamy, Hexagonal image processing: A practical approach, Springer, 2006.

[11] J Serra, "Introduction to mathematical morphology," Computer vision, graphics, and image processing, vol. 35, no. 3, pp. 283-305, 1986.

[12] M Knaup et al., "Ct image reconstruction using hexagonal grids," in IEEE NSS'07. IEEE, 2007, vol. 4, pp. 3074-3076.

[13] J A Fessler and A O Hero, "Space-alternating generalized expectationmaximization algorithm," IEEE TSP, vol. 42, no. 10, pp. 2664-2677, 1994.

[14] M Aharon et al., "K-svd: An algorithm for designing overcomplete dictionaries for sparse representation," IEEE TSP, vol. 54, no. 11, pp. 4311-4322, 2006

[15] J Mairal et al., "Online learning for matrix factorization and sparse coding," JMLR, vol. 11, pp. 19-60, 2010. 